\title{
Origin of volatile element depletion among carbonaceous chondrites
}

\author{
JAN L HELLMANN ${ }^{1}$, TIMO HOPP ${ }^{2}$, CHRISTOPH \\ BURKHARDT $^{1}$ AND THORSTEN KLEINE $^{1}$ \\ ${ }^{1}$ University of Münster, Institut für Planetologie \\ ${ }^{2}$ Origins Laboratory, Department of the Geophysical Sciences \\ and Enrico Fermi Institute
}

Presenting Author: jan.hellmann@uni-muenster.de

Compared to the composition of CI chondrites and the Sun, all other carbonaceous chondrites (CCs) are variably depleted in volatile elements. The origin of this volatile element fractionation remains debated and various models have been proposed. These include the incomplete condensation of volatile elements from the solar nebula [e.g., 1] and variable mixing of volatile-rich and volatile-poor chondrite components [e.g., 2]. To better understand the processes that led to the fracationation of volatile elements, we investigated the mass-dependent Te isotope fractionation among CCs. Tellurium is well suited for this task because as a volatile element with a $50 \%$ condensation temperature $\left(50 \% T_{\mathrm{C}}\right)$ of $\sim 700 \mathrm{~K}$ [3] it is sensitive to isotope fractionation during a variety of nebular processes. We developed a ${ }^{123} \mathrm{Te}^{125} \mathrm{Te}$ double spike method for precise measurements of Te isotope variations by multi-collector ICPMS and obtained mass-dependent Te isotope compositions and Te concentrations for a comprehensive set of samples from the major CC groups.

The CCs exhibit well-resolved inter-group Te isotope variations, with a trend towards lighter isotope compositions for increasingly volatile-depleted samples. Moreover, the Te isotope compositions and concentrations are correlated with the mass fraction of matrix and with nucleosynthetic $\varepsilon^{54} \mathrm{Cr}$ anomalies. Abundances of other volatile elements with $50 \% T_{\mathrm{C}}$ below $\sim 750$ $\mathrm{K}$ are also correlated with the amount of matrix and their relative abundances are CI-chondritic in chondrules/chondrule precursors. Together, these correlations indicate mixing of volatile-rich, isotopically heavy, and ${ }^{54} \mathrm{Cr}$-rich CI-like dust (the matrix) with volatile-poor, isotopically light, and ${ }^{54} \mathrm{Cr}$-poor chondrules/chondrule precursors in variable proportions. Our new data reveal that all CCs contain CI-like dust, that chondrules/chondrule precursors and matrix formed in distinct regions of the disk, and that volatile elements in CCs not only derived from CI-like matrix but also from chondrules/chondrule precursors. As such, CI-like volatile element ratios in Earth's mantle may reflect accretion of volatile-depleted carbonaceous chondrite-like material and do not require accretion of $\mathrm{CI}$ chondrites themselves.

References: [1] Wasson \& Chou (1974) Meteoritics, 9, 69-84. [2] Larimer \& Anders (1967) GCA, 31, 1239-1270. [3] Wood et al. (2019) Am. Mineral., 104, 844-856. 